Full Length Article

\title{
The effects of volar locking plates for distal radius fractures on the image quality of high-resolution peripheral quantitative computed tomography
}

\author{
Makoto Era $^{\mathrm{a}}$, Ko Chiba ${ }^{\mathrm{a}, *}$, Yuichiro Nishino ${ }^{\mathrm{a}}$, Narihiro Okazaki ${ }^{\mathrm{a}}$, Takashi Miyamoto ${ }^{\mathrm{a}}$, \\ Akihiko Yonekura $^{\mathrm{a}}$, Masato Tomita ${ }^{\mathrm{a}}$, Toshiyuki Tsurumoto ${ }^{\mathrm{b}}$, Makoto Osaki ${ }^{\mathrm{a}}$ \\ a Department of Orthopedic Surgery, Nagasaki University Graduate School of Biomedical Sciences, Japan \\ ${ }^{\mathrm{b}}$ Department of Macroscopic Anatomy, Nagasaki University Graduate School of Biomedical Sciences, Japan
}

\section{A R T I C L E I N F O}

\section{Keywords:}

High-resolution peripheral quantitative computed tomography (HR-pQCT)

Fracture healing

Volar locking plate

Image quality

\begin{abstract}
A B S T R A C T
Introduction: High-resolution peripheral quantitative computed tomography (HR-pQCT) has enabled us to observe changes of bone microstructure during fracture healing. However, a method of analyzing the healing process after osteosynthesis has yet to be established due to implant artifacts. The purpose of this study was to investigate the effects of volar locking plates for distal radius fractures on the image quality of HR-pQCT.

Methods: Four different types of plates for distal radius fractures were evaluated. The scan region of HR-pQCT was the center of each plate, not including the screw insertion sites.

To assess plate-generated noise, each plate was fixed to the top of a water-filled rubber glove, scanned by HRpQCT, and the signal-to-noise ratio (SNR) of the region under the plates was calculated. To investigate accuracy, 12 cadaveric radii with and without each plate were scanned by HR-pQCT, and differences between the measured values with and without the plate were evaluated. Differences between the measurements of the entire circumference and of the dorsal third of the radius were also compared. Reproducibility of the in vivo measurement was investigated by repeated scans of 10 patients with distal radius fractures who had undergone surgery with a volar locking plate.

Results: The SNR was significantly higher away from the plate than immediately below the plate. Percentage differences of the measurement values between with and without the plate were $1.4 \%-3.2 \%$ for cortical bone mineral density (Ct.BMD) and 7.2\%-9.8\% for cortical bone thickness (Ct.Th) when the entire circumference was measured. When the dorsal third was measured, they were $0.3 \%-1.7 \%$ for Ct.BMD and $1.8 \%-2.7 \%$ for $\mathrm{Ct}$.Th. The root-mean-square coefficient of variation (RMS\%CV) was $1.12 \%$ for Ct.BMD and 4.18\% for Ct.Th.

Conclusions: The accuracy and reproducibility of cortical bone measurements with a volar locking plate on HRpQCT were acceptable when the dorsal third of the Ct.BMD was analyzed, and this method would be useful for in vivo analysis of the fracture healing process after osteosynthesis.
\end{abstract}

\section{Introduction}

Fracture healing consists of inflammatory, repair, and remodeling phases [1]. In the inflammatory phase, growth factor migration and capillary angiogenesis occur. In the repair phase, membranous ossification and endochondral ossification cause soft callus followed by hard callus formation. In the remodeling phase, natural correction takes place. Previous basic studies of these fracture healing processes have been performed using animal models, with no in vivo analyses in humans.

Fracture healing of patients can be evaluated by plain X-rays and clinical computed tomography (CT). However, plain X-rays provide two-dimensional images that do not enable detailed analysis of fracture healing. Clinical CT enables three-dimensional evaluation, but it can only provide the macrostructure of the cortical bone at the fracture site due to its limited resolution.

High-resolution peripheral quantitative CT (HR-pQCT) is a quantitative CT method for human extremities that provides the highest available resolution (voxel size $61 \mu \mathrm{m}$ ) of any clinical imaging modalities [2-4]. Previously, analysis of human bone microstructure in vivo had required sample collection by invasive techniques such as bone biopsy, which could not be used for longitudinal observations over

\footnotetext{
* Corresponding author at: Department of Orthopedic Surgery, Nagasaki University Graduate School of Biomedical Sciences, 1-7-1, Sakamoto, Nagasaki 852-8501, Japan.

E-mail address: kohchiba@estate.ocn.ne.jp (K. Chiba).
} 

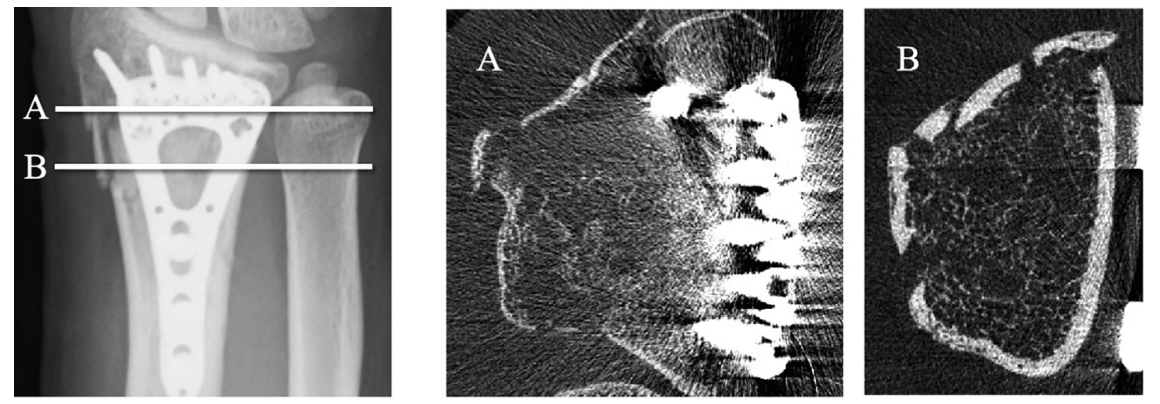

Fig. 1. There are severe metal artifacts at the screw insertion site (A), but these artifacts are not so severe at the center of the plate where no screw is present (B).

time. The advent of HR-pQCT has enabled observation of the changes of human bone microstructure in vivo over time with low radiation exposure.

In previous studies by HR-pQCT, the healing process of distal radius fractures under conservative treatment was observed [5,6]. It has been thought that CT analysis after surgical treatment with implants is challenging due to metal artifacts [7]. Thus, a method of analyzing the healing process after osteosynthesis by HR-pQCT has yet to be established.

In preliminary experiments, we found that severe metal artifacts were present in bone at screw insertion areas (Fig. 1A), but that images of sufficient quality for observation were obtained from areas without screws (Fig. 1B).

In this study, the effects of volar locking plates on the HR-pQCT images were investigated, with the aim of establishing a method of analyzing the postoperative fracture healing process in vivo.

\section{Methods}

Four types of volar locking plates used for distal radius fractures that have a high share of the American, European, and Japanese markets were investigated (Fig. 2). These were the Acu-Loc2 ${ }^{\circledR}$ Standard Volar Distal Radius Plate (Acumed, Hillsboro, OR, USA) (thickness: $2 \mathrm{~mm}$; composition: Ti6Al4V); the DVR ${ }^{\circledR}$ Anatomic Volar Plating System (Biomet Inc., Warsaw, IN, USA) (thickness $2.5 \mathrm{~mm}$; composition: Ti6Al4V); the Stellar2 ${ }^{\circledR}$ (HOYA Technosurgical Corporation, Tokyo, Japan) (thickness: $2.1 \mathrm{~mm}$; composition: Ti6Al4V); and the Variable Angle LCP ${ }^{\circledR}$ Two-Column Volar Distal Radius Plate 2.4 (Depuy Synthes $\mathrm{GmbH}$, Oberdorf, Switzerland) (thickness: $2 \mathrm{~mm}$, composition: pure titanium).

\subsection{Signal-to-noise ratio (SNR)}

First, the noise generated by the plates was evaluated. Each plate was fixed on top of a water-filled rubber glove and scanned by HRpQCT (Xtreme CT II, Scanco Medical, Brüttisellen, Switzerland) (Fig. 3). The scan region was $10 \mathrm{~mm}$ of the center of the plate that extended proximally from the most proximal point on the distal locking screw hole (Fig. 4). The scan parameters were as follows: voxel size $60.7 \mu \mathrm{m}$, effective energy $60 \mathrm{kVp}$, tube current $1470 \mu \mathrm{A}$, integration time $43 \mathrm{~ms}$, scanning time $2 \mathrm{~min}$, and effective dose $5 \mu \mathrm{Sv}$.

Using the images, regions of interest (ROI) were set to $10 \mathrm{~mm} \times 20 \mathrm{~mm} \times 10 \mathrm{~mm}$ immediately below the plate (ROI-1) and at a distance of $10 \mathrm{~mm}$ from the plate (ROI-2) (Fig. 3). The signal-to-noise ratio (SNR) was calculated by dividing the mean signal value inside the ROI by its standard deviation. Each plate was scanned five times, and the mean SNR values were compared with those of control images scanned without the plate.

\subsection{Accuracy analysis by cadaveric bone}

The effects of the plates on the accuracy of cortical bone measurements were then investigated. Twelve cadaveric radii (all right radii from women aged 61-78 years, mean age $71.7 \pm 4.3$ years) were prepared from the collection of the Department of Macroscopic Anatomy in our facility. The study was approved by our facility's ethics committee (approval number: 15033076).

Each plate was placed in the optimum position on the volar side of the distal radius (Fig. 4). The optimum position was determined based on the plate design and anatomical shape of the radius, such as the pronator fossa and watershed line, and the plates were fixed to the bones with sticky jelly. A 10-mm region extending proximally from the most proximal point of the distal locking screw hole was scanned with

\begin{tabular}{|c|c|c|c|c|}
\hline & $\begin{array}{l}\text { Acu-Loc2 } \\
\text { Standard Volar } \\
\text { Radius Plate }\end{array}$ & $\begin{array}{l}\text { DVR } \\
\text { Anatomic Plate }\end{array}$ & Stellar2 & $\begin{array}{l}\text { VA-TCP } \\
\text { Distal Radius } \\
\text { Plate }\end{array}$ \\
\hline $\begin{array}{l}\text { Manufacturer } \\
\text { Composition } \\
\text { Thickness }\end{array}$ & $\begin{array}{l}\text { Acumed } \\
\text { Ti6A14V } \\
2 \mathrm{~mm}\end{array}$ & $\begin{array}{l}\text { ZimmerBiomet } \\
\text { Ti6Al4V } \\
2.5 \mathrm{~mm}\end{array}$ & $\begin{array}{l}\text { HOYA } \\
\text { Ti6A14V } \\
2.1 \mathrm{~mm}\end{array}$ & $\begin{array}{l}\text { Depuy Synthes } \\
\text { Titanium } \\
2 \mathrm{~mm}\end{array}$ \\
\hline & ) & & & {$\left[\begin{array}{l}000 \\
0 \\
0 \\
0 \\
0 \\
0 \\
0\end{array}\right.$} \\
\hline
\end{tabular}

Fig. 2. Volar locking plates for distal radius fractures investigated in this study. 


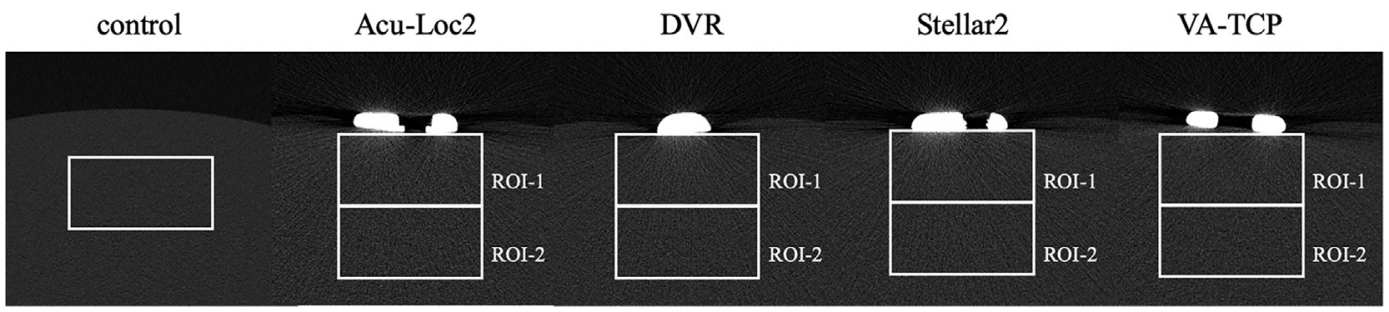

Fig. 3. Each plate is placed on top of a water balloon and scanned by HR-pQCT. ROIs (10 $\mathrm{mm} \times 20 \mathrm{~mm} \times 10 \mathrm{~mm})$ are set immediately below the plate (ROI-1) and at a distance of $10 \mathrm{~mm}$ (ROI-2), and their SNRs are compared.

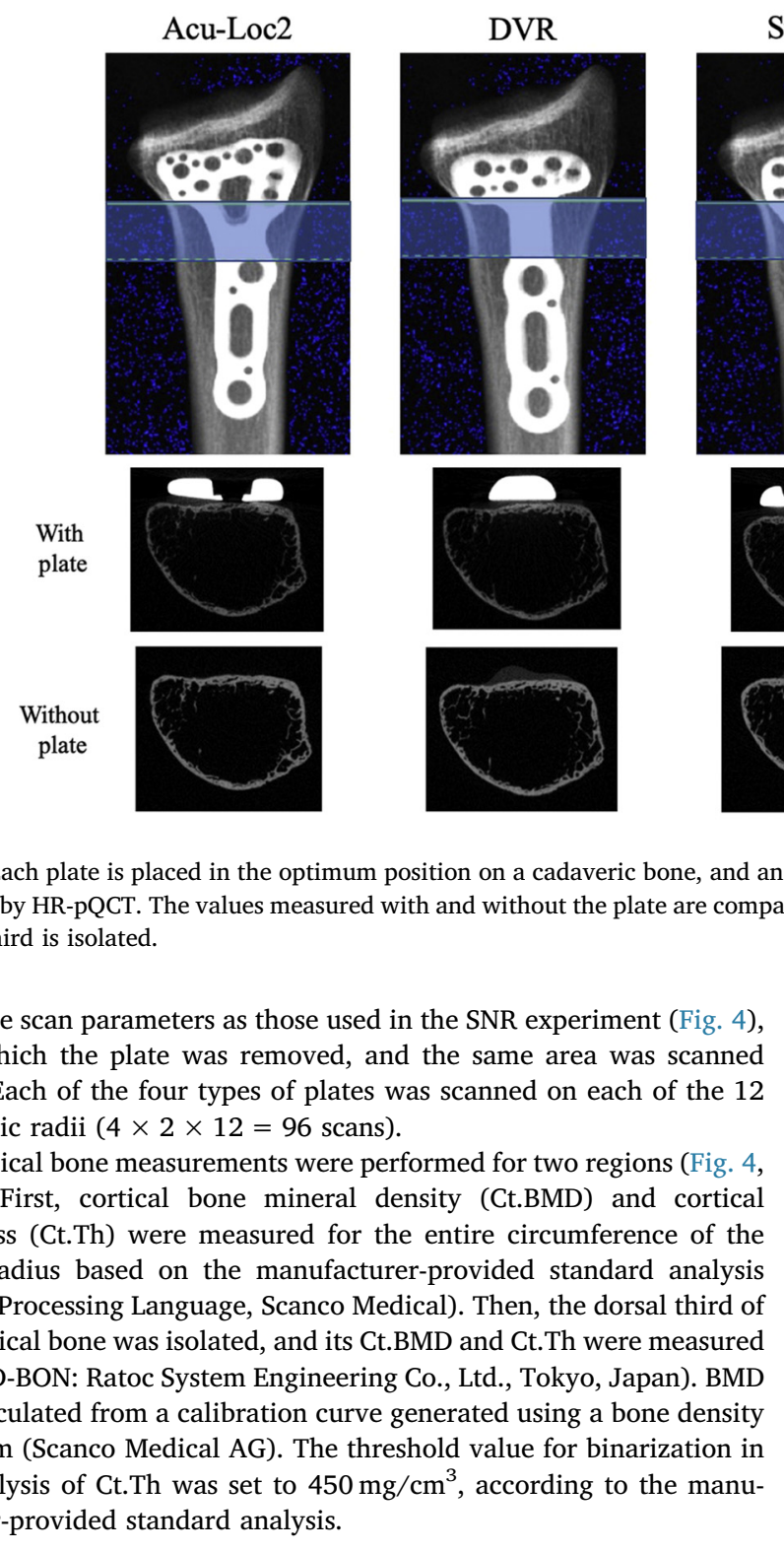

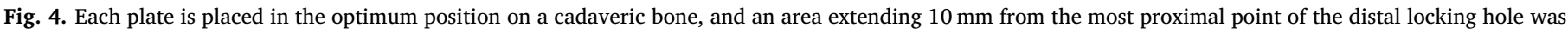

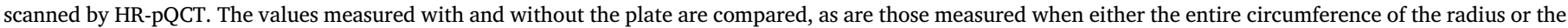
dorsal third is isolated.

the same scan parameters as those used in the SNR experiment (Fig. 4), after which the plate was removed, and the same area was scanned again. Each of the four types of plates was scanned on each of the 12 cadaveric radii $(4 \times 2 \times 12=96$ scans $)$.

Cortical bone measurements were performed for two regions (Fig. 4, right). First, cortical bone mineral density (Ct.BMD) and cortical thickness (Ct.Th) were measured for the entire circumference of the distal radius based on the manufacturer-provided standard analysis (Image Processing Language, Scanco Medical). Then, the dorsal third of the cortical bone was isolated, and its Ct.BMD and Ct.Th were measured (TRI/3D-BON: Ratoc System Engineering Co., Ltd., Tokyo, Japan). BMD was calculated from a calibration curve generated using a bone density phantom (Scanco Medical AG). The threshold value for binarization in the analysis of Ct.Th was set to $450 \mathrm{mg} / \mathrm{cm}^{3}$, according to the manufacturer-provided standard analysis.

\subsection{Reproducibility of in vivo measurements}

Reproducibility was then evaluated in actual patients (Fig. 5). The subjects were 10 patients with distal radius fractures who had undergone osteosynthesis with a variable angle two-column plate (VA-TCP) in the Department of Orthopaedic Surgery (3 men and 7 women; age 21-74 years, mean age $53.9 \pm 14.8$ years; 4 fractures of the right arm and 6 of the left arm). The study was approved by our hospital's clinical research ethics committee (approval number: 16031418), and informed consent was obtained from the participants.
HR-pQCT scanning was performed with the same scan parameters and at the same scan site as those used in the cadaver experiment, twice without repositioning (Fig. 5), and Ct.BMD and Ct.Th at the dorsal third of the cortical bone were measured.

To measure precisely the same region in the two different scans, measurements were performed as below: 1) two images were matched three-dimensionally; 2) the dorsal third of the cortical bone was extracted; 3) upper and lower 10\% (total 20\%) heights of the cortical bone were excluded; and 4) Ct.BMD and Ct.Th were measured at the

\subsection{Statistical analysis}

A paired $t$-test was used for comparisons between ROI-1 and ROI-2 in the SNR experiments, and Tukey-Kramer's honestly significant difference (HSD) test was used for comparisons between control and plates, with $p<0.01$ regarded as significant (JMP ${ }^{\circledR}$ Pro 13, SAS Institute Inc., Cary, NC, USA).

In the cadaver study, percentage differences in values measured with and without the plates were calculated, and the rates when the entire circumference of the radius was measured were compared with those when only the dorsal third was isolated and measured. A mixed effects model was used for statistical analysis, with $p<0.01$ regarded as significant (JMP ${ }^{\circledR}$ Pro 13, SAS Institute Inc., Cary, NC, USA).

In the in vivo precision study, reproducibility was evaluated using the root-mean-square coefficient of variation (RMS\%CV) [8]. RMS\%CV middle $80 \%$ region. 


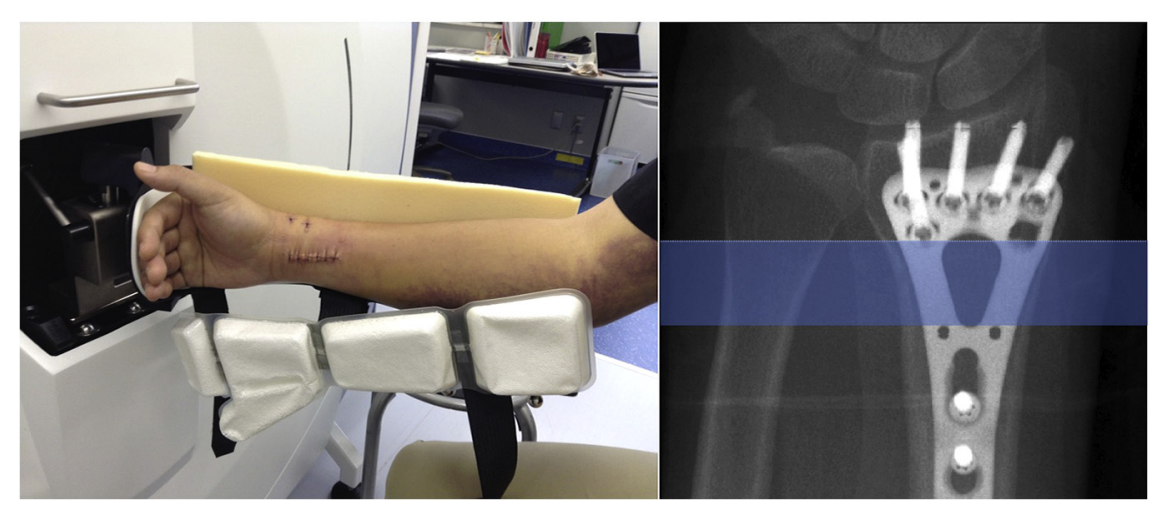

Fig. 5. Patients with a distal radius fracture treated with a volar locking plate (VA-TCP) were scanned by HR-pQCT twice, with the BMD and thickness measured at the dorsal third of the cortical bone.

Table 1

SNR at ROI-1 and ROI-2 in each plate.

\begin{tabular}{llllll}
\hline Control & & Acu-Loc2 & DVR & Stellar2 & \multicolumn{1}{c}{ VA-TCP } \\
\hline $43.3 \pm 0.7$ & ROI-1 & $33.4 \pm 0.7^{* *}$ & $34.9 \pm 1.5^{*}$ & $32.3 \pm 0.8^{* * *}$ & $33.3 \pm 0.7^{* * *}$ \\
& ROI-2 & $39.6 \pm 0.5^{* *} \dagger$ & $41.5 \pm 1.1^{*} \dagger$ & $40.5 \pm 0.3^{* *} \dagger$ & $39.9 \pm 0.9^{* *} \dagger$ \\
\hline
\end{tabular}

Comparison between the control and each plate using Tukey-Kramer's HSD test. ${ }^{*} p<0.01,{ }^{* *} p<0.001$.

Comparison between ROI-1 and ROI-2 using a paired $t$-test. $\dagger p<0.001$.

Table 2

Measurement values with and without each plate and their percentage differences.

\begin{tabular}{|c|c|c|c|c|c|c|c|c|}
\hline & & \multicolumn{3}{|l|}{ Whole } & \multicolumn{3}{|l|}{$1 / 3$ dorsal } & \multirow[t]{2}{*}{$P$ value } \\
\hline & & Without plate & With plate & $\%$ & Without plate & With plate & $\%$ & \\
\hline \multirow{4}{*}{$\begin{array}{l}\text { Ct.BMD } \\
\qquad\left(\mathrm{mg} / \mathrm{cm}^{3}\right)\end{array}$} & Acu-Loc2 & $979.7 \pm 49.8$ & $990.5 \pm 46.5$ & +1.4 & $936.5 \pm 34.7$ & $928.0 \pm 49.5$ & -1.3 & 0.82 \\
\hline & DVR & $957.2 \pm 82.5$ & $961.9 \pm 52.5$ & +3.2 & $906.8 \pm 72.3$ & $913.9 \pm 59.5$ & +1.7 & 0.54 \\
\hline & Stellar2 & $985.9 \pm 55.1$ & $1014.6 \pm 49.5$ & +3.1 & $940.0 \pm 35.2$ & $940.3 \pm 36.7$ & +0.3 & $<0.01$ \\
\hline & VA-TCP & $998.8 \pm 47.5$ & $992.8 \pm 44.5$ & -1.9 & $947.3 \pm 32.8$ & $944.1 \pm 33.9$ & -0.4 & $<0.01$ \\
\hline \multirow{4}{*}{$\begin{array}{l}\text { Ct.Th } \\
\text { (mm) }\end{array}$} & Acu-Loc2 & $0.79 \pm 0.10$ & $0.86 \pm 0.10$ & +8.3 & $0.63 \pm 0.07$ & $0.64 \pm 0.07$ & +2.7 & $<0.001$ \\
\hline & DVR & $0.79 \pm 0.09$ & $0.84 \pm 0.09$ & +8.5 & $0.61 \pm 0.07$ & $0.62 \pm 0.07$ & +1.8 & $<0.001$ \\
\hline & Stellar2 & $0.80 \pm 0.09$ & $0.88 \pm 0.09$ & +9.8 & $0.63 \pm 0.07$ & $0.65 \pm 0.06$ & +2.6 & $<0.001$ \\
\hline & VA-TCP & $0.82 \pm 0.09$ & $0.88 \pm 0.09$ & +7.2 & $0.66 \pm 0.07$ & $0.67 \pm 0.06$ & +2.3 & $<0.001$ \\
\hline
\end{tabular}

Ct.BMD: cortical bone mineral density, Ct.Th: cortical thickness.

Comparison of the percent differences between the whole circumference and the 1/3 dorsal region using mixed effect model.

was calculated using the following formula.

$R M S \% C V=\sqrt{ } \frac{1}{n} \sum(100 * S D / \text { mean })^{2}$

\section{Results}

Table 1 shows the results of the SNR analysis using a water-filled rubber glove with a plate (Fig. 3). The SNR was significantly lower at the ROIs in each plate than the control value. For every plate, the SNR at ROI-2, located away from the plate, was significantly higher than that at ROI-1, located close to the plate.

Table 2 shows the percentage changes of cortical bone measurements with and without the plate on the cadaveric distal radii (Fig. 4). When the entire circumference of the radius was analyzed, the percentage change in measurements was $1.4 \%-3.2 \%$ for Ct.BMD and $7.2 \%-9.8 \%$ for Ct.Th. When the dorsal third was isolated and analyzed, the percentage change was only $0.3 \%-1.7 \%$ for Ct.BMD and $1.8 \%-2.7 \%$ for Ct.Th.

There was no significant difference in the percentage differences of the measured values due to each type of plate, but the difference was larger for Ct.Th than for Ct.BMD. When the dorsal third of the radius was analyzed, the percentage change was significantly smaller than that for the entire circumference of the radius.
Examining the reproducibility of in vivo cortical bone measurements, the mean Ct.BMD measured in the dorsal third of the radius bones of 10 patients with distal radius fractures was $816.4 \pm 111.1 \mathrm{mg} / \mathrm{cm}^{3}\left(689.5-988 \mathrm{mg} / \mathrm{cm}^{3}\right)$, and the mean Ct.Th was $0.83 \pm 0.35 \mathrm{~mm}(0.01-1.37 \mathrm{~mm})$; the RMS $\% \mathrm{CV}$ was $1.12 \%$ for Ct.BMD and $4.18 \%$ for Ct.Th.

Fig. 6 shows a case with a distal radial fracture treated with a volar locking plate (VA-TCP). An increase of cortical BMD of the fracture site at the dorsal cortical bone can be observed by the repeated scans and measurements after osteosynthesis.

\section{Discussion}

In the present study, the effects of volar locking plates for distal radius fractures on HR-pQCT image quality were investigated.

The plates scanned in this study consisted of either titanium or a titanium alloy (Fig. 2). Titanium shows lower X-ray absorbance and is reportedly less likely to cause metal artifacts than is stainless steel, which was formerly used as an implant material $[9,10]$. The results of the present study indicated that artifacts had less effect in ROIs located away from the plate (Tables 1,2 ). If the entire circumference is analyzed, the ROI in cortical bone on the volar side will include the area immediately below the plate, which is severely affected by metal 

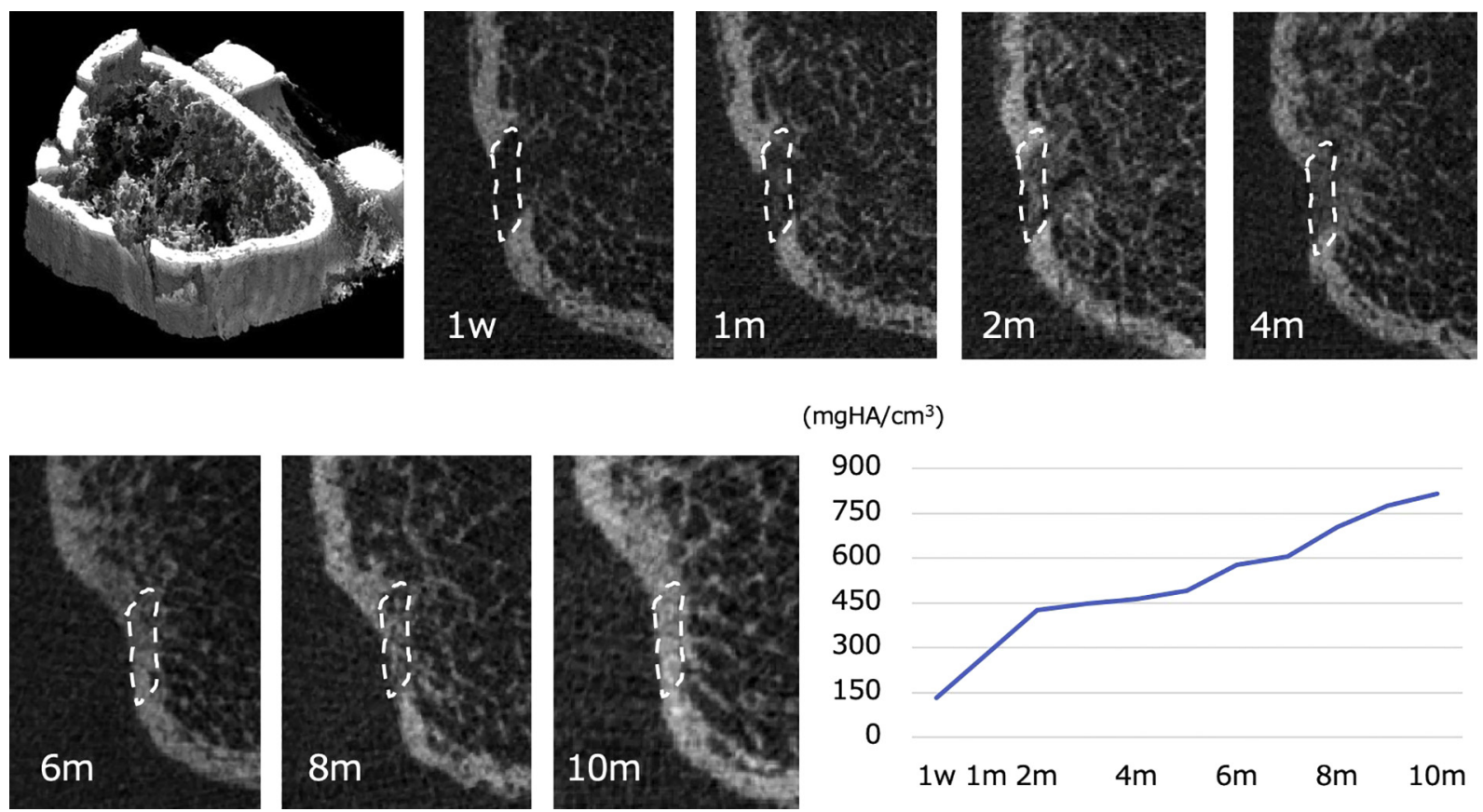

$\left(\mathrm{mgHA} / \mathrm{cm}^{3}\right)$

Fig. 6. A patient with a distal radius fracture treated with a volar locking plate (VA-TCP) was periodically scanned by HR-pQCT. Cortical BMD at the dorsal fracture site increases gradually with time.

artifacts, resulting in a greater difference in the measured value of cortical bone thickness in particular.

De Jong et al. wrapped 15 cadaver radial bones with a plaster-ofParis or a fiberglass cast, scanned them with HR-pQCT, and investigated the effect of the cast material on HR-pQCT measurements [11]. They found that the percentage differences in measurements were $-5.1 \%$ for Ct.BMD and $-9.8 \%$ for Ct.Th with the plaster-of-Paris cast and $-0.9 \%$ for Ct.BMD and $-1.6 \%$ for Ct.Th with the fiberglass cast.

De Jong et al. also performed a pilot study using a single fresh frozen forearm with plates of three different materials [carbon-fiberreinforced poly-ether-ether-ketone (CFR-PEEK), Ti6Al4V, and Ti6Al7Nb], scanned them with HR-pQCT, and analyzed their effects on the bone microstructure parameters of the entire circumference of the radius [12]. The percentage differences due to CFR-PEEK were $-1.6 \%$ for Ct.BMD and $-3.2 \%$ for Ct.Th, those due to Ti6Al4V were $+19.6 \%$ for Ct. BMD and $+27.2 \%$ for Ct.Th, and those due to Ti6Al7Nb were $-0.3 \%$ for Ct.BMD and $+0.7 \%$ for Ct.Th. Although CFR-PEEK is commercially available in Europe and North America, it is not yet marketed in Japan, meaning that it is not yet possible to carry out studies of this implant in this country. A comparison of accuracy for Ti6Al4V, which was also used in this study, suggested that our method of isolating and analyzing the dorsal third of the bone is more accurate than analyzing the entire circumference.

Reproducibility was good for Ct.BMD, for which the RMS\%CV was $1.13 \%$, suggesting that this method may be usable in clinical studies analyzing the fracture healing process. On the other hand, RMS\%CV of Ct.Th was $4.18 \%$, showing that in vivo analysis of the cortical bone microstructure is still challenging.

De Jong et al. also carried out an investigation of reproducibility by fitting 16 healthy human volunteers with fiberglass casts and performing HR-pQCT scanning. The RMS-CV was $0.47 \%$ for Ct.BMD and $0.90 \%$ for Ct.Th. In a study of CFR-PEEK, Ti6Al4V, and Ti6Al7Nb plates attached to a single fresh frozen forearm, the RMS\%CV was $1.48 \%$ for Ct.BMD and $1.55 \%$ for Ct.Th with the CFR-PEEK plate, $1.13 \%$ for Ct.BMD and $1.65 \%$ for Ct.Th with the Ti6Al4V plate, and $0.06 \%$ for Ct.BMD and $0.00 \%$ for Ct.Th with the Ti6Al7Nb plate. The reproducibility of Ct.BMD in the present study was thus acceptable compared with those previous studies.

\subsection{Limitations}

This study had several limitations. The first was that scanning could only be carried out at the center of the plate, and the presence of screws prevented evaluation of the distal region where major fractures are located (Fig. 1). This method is only for use in studies of fracture healing, and it cannot be used for the clinical evaluation of synostosis. Second, the evaluation site proposed in the present study was only the dorsal third of the cortical bone (Fig. 4). Evaluation of the cortical bone around the metal plate was not recommended to avoid inaccurate measurement. Third, because the cadaveric bone and the osteoporosis patients in this study contained very little trabecular bone at the scanned site (Fig. 4), trabecular bone parameters could not be verified. Lastly, each experiment included only a small number of subjects.

\section{Conclusions}

The effects of volar locking plates on HR-pQCT images were investigated to establish an in vivo analysis method of the fracture healing process after surgical treatment. The accuracy and reproducibility of our method of evaluating the dorsal third of Ct.BMD were within acceptable limits, making this method useful for analyzing the fracture healing process after osteosynthesis.

\section{Declaration of Competing Interest}

The authors declare no conflicts of interest associated with this manuscript.

\section{Acknowledgments}

The authors would like to thank Shuntaro Sato for advising on the statistical analysis.

\section{References}

[1] Ruedi TP, Buckley RE, Moran CG. AO Principles of Fracture Management Second expanded edition.

[2] A.J. Burghardt, T.M. Link, S. Majumdar, High-resolution computed tomography for 
clinical imaging of bone microarchitecture, Clin. Orthop. Relat. Res. 469 (8) (2011) 2179-2193.

[3] K.K. Nishiyama, E. Shane, Clinical imaging of bone microarchitecture with HRpQCT, Curr. Osteoporos. Rep. 11 (2) (2013) 147-155.

[4] A.M. Cheung, J.D. Adachi, D.A. Hanley, et al., High-resolution peripheral quantitative computed tomography for the assessment of bone strength and structure: review by the Canadian Bone Strength Working Group, Curr. Osteoporos. Rep. 11 (2) (2013) 136-146.

[5] J.J.A. de Jong, P.C. Willems, J.J. Arts, et al., Assessment of the healing process in distal radius fractures by high resolution peripheral quantitative computed tomography, Bone 64 (2014) 65-74 Jul.

[6] U. Meyer, J.J. de Jong, S.G.P. Bours, et al., Early changes in bone density, microarchitecture, bone resorption, and inflammation predict the clinical outcome 12 weeks after conservatively treated distal radius fractures: an exploratory study, $\mathrm{J}$. Bone Miner. Res. 29 (9) (2014) 2065-2073 Sep.

[7] M.J. Lee, S. Kim, S.A. Lee, et al., Overcoming artifact from metallic orthopedic implants at high-field-strength MR imaging and multi-detector CT, RadioGraphics
27 (3) (2007) 791-803 May-Jun.

[8] K. Chiba, N. Okazaki, A. Kurogi, et al, Precision of second-generation high-resolution peripheral quantitative computed tomography: intra- and intertester reproducibilities and factors involved in the reproducibility of cortical porosity, $\mathrm{J}$. Clin. Densitom. 21 (2) (2018) 295-302 Jun.

[9] J.F. Barrett, N. Keat, Artifacts in CT: recognition and avoidance, Radiographics 24 (6) (2004) 1679-1691 Nov-Dec.

[10] M.-J. Lee, S. Kim, S.-A. Lee, et al., Overcoming artifacts from metallic orthopedic implants at high-field-strength MR imaging and multi-detector CT, Radiographics 27 (3) (2007) 791-803 May-Jun.

[11] J.J.A. de Jong, J.J. Arts, U. Meyer, et al., Effect of a cast on short-term reproducibility and bone parameters obtained from HR-pQCT measurements at the distal end of the radius, J. Bone Joint Surg. 98-A (2016) 356-362 Mar.

[12] J.J.A. de Jong, A. Lataster, B. van Rietbergen, et al., Distal radius plate of CFR-PEEK has minimal effect compared to titanium plates on bone parameters in high-resolution peripheral quantitative computed tomography: a pilot study, BMC Med. Imaging 17 (2017) 18. 